\title{
A Navigation Method for Land Vehicles Based on DR with Computer Vision Aided
}

\author{
Xingyu $\mathrm{Li}^{1, \text { a }}$, Tingting Jiang ${ }^{2, \mathrm{~b}}$ and Zhenguo Yuan ${ }^{3, \mathrm{c}}$ \\ ${ }^{1}$ Heilongjiang University, Harbin 150080, China; \\ ${ }^{2}$ Heillongjiang Polytechnic University, Harbin 150080, China; \\ ${ }^{3}$ GUANGDONG INDUSTRY TECHNICAL COLLEGE, Guangzhou 510300, China. \\ aLiXingyug@hotmail.com, bittingof@sina.com, cyuanzhenguodaohang@163.com
}

Keywords: Computer vision, DR, Land vehicle, Navigation.

\begin{abstract}
Visual navigation methods become more and more hot issues for the vehicles. Computer vision has been applied to Dead Reckoning (DR) for land vehicles successfully. Based on the principle of DR, computer vision is used to calculate the azimuth information. And displacement information from camera could also be a good reference for DR. The navigation method reduces the cost and improves the accuracy. Results show that the navigation method can improve the position accuracy for land vehicle.
\end{abstract}

\section{Introduction}

DR is a commonly used navigation method for land vehicles. The accuracy of DR is high in a short time but decreases over time [1]. Taking DR as the only navigation method is not a good way for land vehicle. Global Positioning System (GPS) is also a means of navigation and it can correct the error of DR, which makes good compensation for poisoning [2]. However, it is cumbersome for GPS to achieve stable and high-level navigation in complex environment. A novel navigation way for land vehicle using DR and computer vision is discussed in this paper.

With the development of computer application, the processing speed of visual navigation system has been greatly improved. The principle of visual navigation system is shown in figure 1[3-4]:

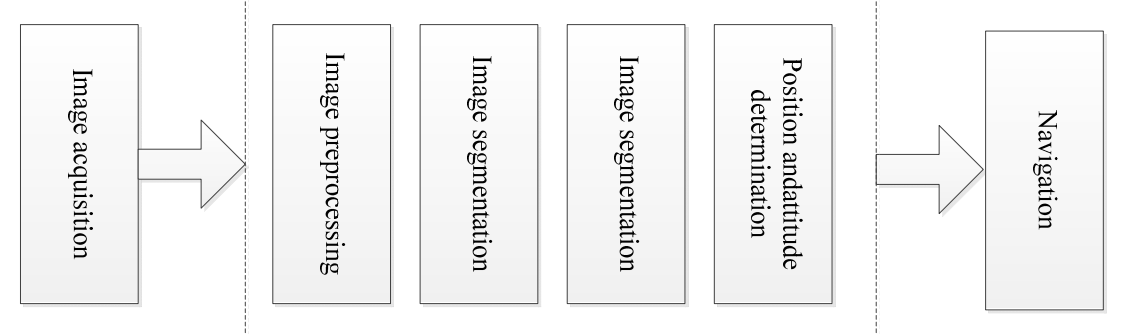

Fig. 1 Principle of visual navigation system

Visual navigation system uses camera to collect the image of the target and navigates by means of obtaining characteristic information of the object. The process of visual navigation system contains image preprocessing, image segmentation, image segmentation and position and attitude determination. The last step of the process will be emphatically studied for land vehicle navigation.

Computer vision employs computer and its related equipment to analog biological vision. Its main task is to obtain the corresponding scene information by the picture or video capture. The final goal of computer vision is to make computer observe and understand the world, which is a long-range goal. Certain task can still be accomplished before realizing the final target. With the development of camera technology and the widely use of camera automobile data recorder, it is more likely to use computer vision aiding DR for land-vehicle navigation. Based on the principle of DR, the algorithm of computer vision aiding DR is as follows. 


\section{Principle of DR for Land Vehicles}

Generally, the movement of vehicles is approximately seen as two-dimensional motion on the earth surface plane. If the initial position and displacement at each moment is known, the vehicle position can be calculated by displacement accumulation. The vehicle position can be descried in East and North coordinate system. If the sampling rate is high enough, the travel distance of the vehicle in two adjacent sampling intervals will be treated as linear displacement. The schematic diagram of DR principle is shown in figure 2[5]:

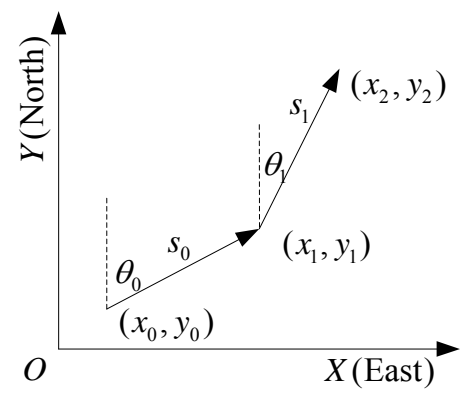

Fig. 2 Schematic diagram of DR principle

The initial position of the vehicle is $\left(x_{0}, y_{0}\right)$. The position of next time is $\left(x_{1}, y_{1}\right)$ :

$\left\{\begin{array}{l}x_{1}=x_{0}+s_{0} \cdot \sin \theta_{0} \\ y_{1}=y_{0}+s_{0} \cdot \cos \theta_{0}\end{array}\right.$

Where $s_{0}$ is the displacement, $\theta_{0}$ is the angle between the driving direction and North. So $\left(x_{2}, y_{2}\right)$ is:

$$
\left\{\begin{array}{l}
x_{2}=x_{1}+s_{1} \cdot \sin \theta_{1}=x_{0}+\left(s_{0} \cdot \sin \theta_{0}+s_{1} \cdot \sin \theta_{1}\right) \\
y_{2}=y_{1}+s_{1} \cdot \cos \theta_{1}=y_{0}+\left(s_{0} \cdot \cos \theta_{0}+s_{1} \cdot \cos \theta_{1}\right)
\end{array}\right.
$$

So the position at moment $k$ :

$$
\left\{\begin{array}{l}
x_{k}=x_{0}+\sum_{i=0}^{k} s_{i} \cdot \sin \theta_{i} \\
y_{k}=y_{0}+\sum_{i=0}^{k} s_{i} \cdot \cos \theta_{i}
\end{array}\right.
$$

From Equation (3), the heading angle and the displacement are needed to obtain the position information. Angular rate gyroscope can calculate the heading angle. The output of the angular rate gyroscope is the bearings changing rate of the vehicle. However, constant error and drift error exist in the gyroscope, which will make error. And the error grows larger with the integration. So the angular rate gyroscope is inconformity for long-time use and the accuracy of DR decreases. Accelerometer can record the displacement. The displacement can be got from velocity integral and the velocity will be obtained from acceleration integral. Because of the zero-bias, the error will also grow with time. So another extra navigation system is needed to aid DR.

\section{Auxiliary Principle of Computer Vision}

Computer vision navigation obtains information of alignment parameter by vision sensor. It deals with the graphic information and has advantages of completely independence and passivity. The main purpose of computer vision aiding DR is calculating the heading angle and the displacement by computer vision. Then use algorithm of Dead Reckoning to provide navigation information [6].

\section{Common Coordinate System.}

The coordinate system for computer vision contains global coordinate system, camera coordinate system and image coordinate system commonly.

Global coordinate system is three-dimensional coordinate system delimited by user. The object to be measured and the camera are seem as an ensemble and the optic center of the camera is the 
coordinate center. Camera coordinate system also uses optic center of the camera as coordinate center, the Photography direction is forward direction, the horizontal coordinate is parallel with physical coordinate. The image coordinate system, $\mathrm{z}$ direction is coincide with optical axis of camera, $\mathrm{x}$ and $\mathrm{y}$ direction are parallel with physical coordinate.

\section{Heading Angle Calculation.}

The coordinate of the object in global coordinate system is , while is in camera coordinate system. The transformation relation between two coordinates is [7]:

$$
\left[\begin{array}{l}
x_{c} \\
y_{c} \\
z_{c}
\end{array}\right]=\left[\begin{array}{ccc}
\cos \theta \sin \psi \sin \phi-\sin \theta \cos \phi & \sin \psi \sin \phi+\cos \theta \cos \phi & \cos \psi \sin \phi \\
\cos \theta \sin \psi \cos \phi+\sin \theta \sin \phi & \sin \theta \sin \psi \cos \phi & \cos \psi \cos \phi \\
\cos \theta \cos \psi & \sin \theta \cos \psi & -\sin \psi
\end{array}\right]\left[\begin{array}{c}
x_{g} \\
y_{g} \\
z_{g}
\end{array}\right]+\left[\begin{array}{c}
O_{x} \\
O_{y} \\
O_{z}
\end{array}\right]
$$

Where $\theta, \psi, \phi$ is heading angle, pitch angle, roll angle respectively. $\left[\begin{array}{lll}O_{x} & O_{y} & O_{z}\end{array}\right]^{T}$ is the origin of global coordinate system in the camera coordinate system.

The coordinate of the object in camera coordinate system has to be changed into image physical coordinate system:

$$
\left\{\begin{array}{l}
X=f x_{c} / z_{c} \\
Y=f y_{c} / z_{c}
\end{array}\right.
$$

Where $f$ is focal length of the camera, image pixel point is $\left(\begin{array}{ll}u & w\end{array}\right),\left(\begin{array}{ll}u_{0} & w_{0}\end{array}\right)$ is image center. $S_{x}, S_{y}$ is sampling frequency of $X, Y$ direction.

$$
\left\{\begin{array}{l}
u-u_{0}=S_{x} X \\
w-w_{0}=S_{y} Y
\end{array}\right.
$$

From equation (5) and (6), derivation can be obtained:

$$
\left\{\begin{array}{l}
u-u_{0}=f S_{x} x_{c} / z_{c} \\
w-w_{0}=f S_{y} y_{c} / z_{c}
\end{array}\right.
$$

The heading angle can be calculated:

$$
\theta=\frac{\sin \psi \sin \phi+\cos \theta \cos \phi}{\sin \theta \sin \psi \cos \phi}
$$

\section{Position Calculation.}

The position in global coordinate system transforms into image coordinate system[8]:

$$
Z_{c}\left[\begin{array}{c}
u \\
w \\
1
\end{array}\right]=\left[\begin{array}{ccc}
1 / \omega_{x} & 0 & u_{0} \\
0 & 1 / \omega_{y} & v_{0} \\
0 & 0 & 1
\end{array}\right]\left[\begin{array}{cccc}
f & 0 & 0 & 0 \\
0 & f & 0 & 0 \\
0 & 0 & 1 & 0
\end{array}\right]\left[\begin{array}{cc}
R & T \\
0 & 1
\end{array}\right]\left[\begin{array}{c}
X_{g} \\
Y_{g} \\
Z_{g} \\
1
\end{array}\right]=\left[\begin{array}{cccc}
A_{11} & A_{12} & A_{13} & A_{14} \\
A_{21} & A_{22} & A_{23} & A_{24} \\
A_{31} & A_{32} & A_{33} & 1
\end{array}\right]\left[\begin{array}{c}
X_{g} \\
Y_{g} \\
Z_{g} \\
1
\end{array}\right]
$$

Where $R \in 3 \times 3$ is rotation matrix, $T \in 3 \times 1, \omega_{x}, \omega_{y}$ is physical size of pixel in $X, Y$ direction.

Land vehicle moves in plane motion, $Z_{g}=0$.So equation (9) changes into:

$$
Z_{c}\left[\begin{array}{c}
u \\
w \\
1
\end{array}\right]=\left[\begin{array}{ccc}
A_{11} & A_{12} & A_{14} \\
A_{21} & A_{22} & A_{24} \\
A_{31} & A_{32} & 1
\end{array}\right]\left[\begin{array}{c}
X_{g} \\
Y_{g} \\
1
\end{array}\right]=H\left[\begin{array}{c}
X_{g} \\
Y_{g} \\
1
\end{array}\right]
$$

Let be $H^{-1}=\left[\begin{array}{lll}h_{11} & h_{12} & h_{13} \\ h_{21} & h_{22} & h_{23} \\ h_{31} & h_{32} & h_{33}\end{array}\right]$, the position can be calculated: 


$$
\left\{\begin{array}{l}
X_{g}=\frac{h_{11} u+h_{12} w+h_{13}}{h_{31} u+h_{32} w+h_{33}} \\
Y_{g}=\frac{h_{21} u+h_{22} w+h_{23}}{h_{31} u+h_{32} w+h_{33}}
\end{array}\right.
$$

\section{Experiment and Results}

Car experiment is made, the Initial latitude is $100.909^{\circ}$, the Initial longitude is $41.246^{\circ}$, the locus is shown in figure 3 :

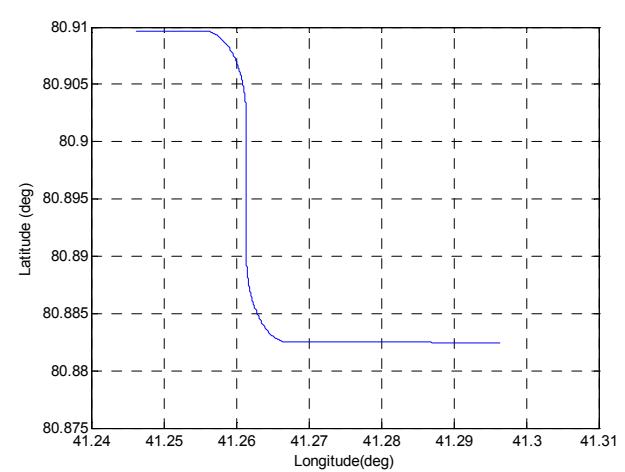

Fig. 3 Motion trajectory

The parameters of DR are as follows: angular rate gyroscope is $0.15 \%$, accelerometer is $5 \mathrm{mg}$, odometer is $0.02 \%$. The position error of the vehicle by DR is shown in figure 4,5:

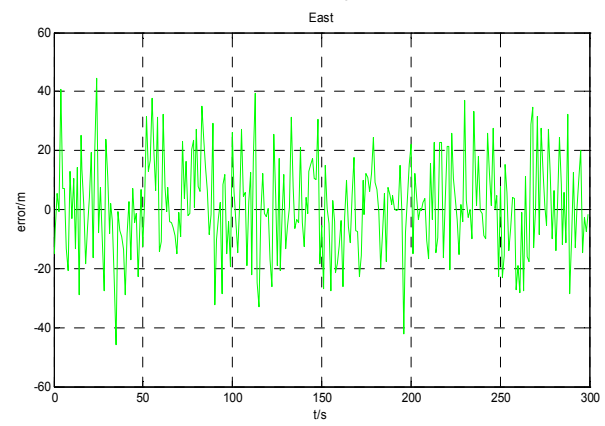

Fig. 4 East position error of DR

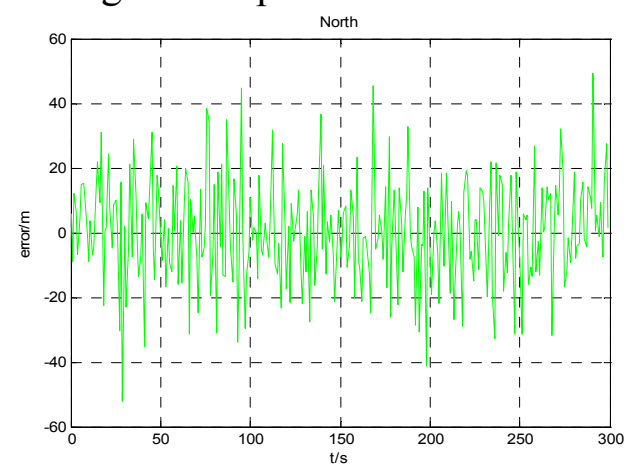

Fig. 5 North position error of DR

Figure 4 and 5 are the position error calculated only by DR. The east error and north error are between $-50 \mathrm{~m}$ to $50 \mathrm{~m}$.

With computer vision aided, the position error is shown in figure 6, 7: 


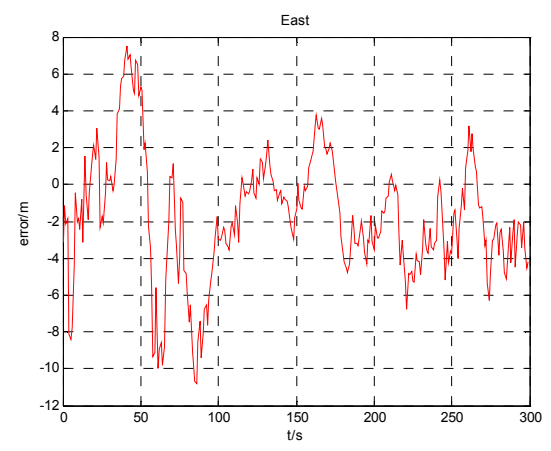

Fig. 6 East position error with computer vision aided

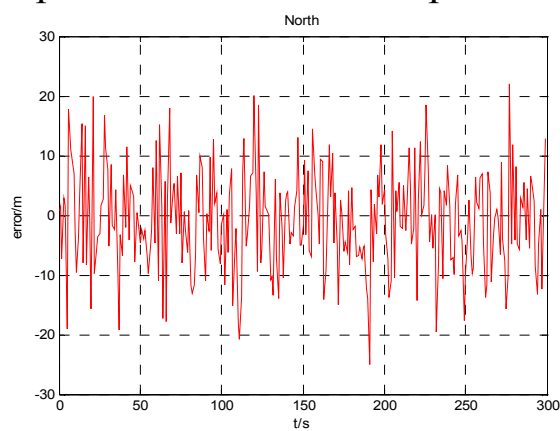

Fig. 7 North position error with computer vision aided

Figure 6 and 7 are the position error calculated by DR and computer vision. The east error and north error are between $-20 \mathrm{~m}$ to $30 \mathrm{~m}$, the east error is obviously smaller. In this paper, the auxiliary computer vision used for land vehicles based on DR is studied, the position accuracy is improved.

\section{References}

[1]. Zhao L, Ochieng W Y, Quddus M A. An extended Kalman filter algorithm for integrating GPS and low cost dead reckoning system data for vehicle performance and emissions monitoring, Journal of Navigation. Vol.56 (2003) No.2, p. 257-275.

[2]. Lin Zhao, Zhenguo Yuan. GPS/DR vehicle integrated navigation system based on Central Difference Kalman Filter, Journal of Information and Computational Science. Vol.9 (2012) No. 10, p. 2771-2779.

[3]. Yong Jiang, Jie Cao, Ruihua Liu, Yaling Du. An Algorithm for Automatic Guided Vehicle Based on Machine Vision and DR. Proceedings of the 6th World Congress on Intelligent Control and Automation .Dalian, China, 2006, June 21- 23 p. 8582-8586.

[4]. Huang Xianlin, Jiang Xiaonan, Lu Hongqian Li Mingming. Survey of Vision for Autonomous Navigation. Journal of Jilin University (InformationScienceEdition), Vol.28 (2010) No.2, p. 158-165.

[5]. Zhang Chuanbin, Tian Wei-feng, Jin Zhihua. Study on nonlinear filtering method for land vehicle DR navigation. Systems Engineering and Elec-tronics, Vol.27 (2005) No.6, p.1058-1060.

[6]. DALGLEISHFR, TETLOWSW, ALLWOODRL. Vision Based Navigation of Unmanned Underwater Vehicles: A Survey Part I: Vision Based Cable-, Pipeline-and Fish Tracking. Proceedings of the Institute of Marine Engineering, Science and Technology. London, England, 2004, p.51-58.

[7]. A. CRIMINISI, I. REID and A. ZISSERMAN. A Plane Measuring Device. Image and Vision Computing.Vol.17 (1999) No.8, p. 625-634. 
[8]. N Kumar, PN Belhumeur, A Biswas, DW Jacobs .Leafsnap: A computer vision system for automatic plant species identification. Computer Vision-ECCV 2012: 12th European Conference on Computer Vision, Florence, Italy, 2012, October 7-13, p.1258-1265. 